cool IR source. However, light can escape through the holes of the donut and there it strikes additional material, presumably also ejected by the central star, which bounces part of the light in our direction. Thus we see two beautiful reflection nebulae in the shape of a cat.

Schmidt and Cohen (Astrophys. J. 246; 444,1981 ) have made detailed spectropolarimetric and spectrophotometric studies of two other bipolar nebulae that resemble GL2688. Dense condensations, and neutral gas in their shadows, are found to fill much of the volume of the nebulae, reinforcing the view that these bipolar objects represent a very early phase in the life of a planetary nebula. Recent models for these bipolar nebulae include biconical outflow above a luminous disk (Icke $A$ strophys. J. 247; 152, 1981) and gravitational ejection of matter from the atmosphere of a red giant star by a close companion star orbiting around it (Morris Astrophys. J., in the press).

Radio astronomers have also studied GL2688 and find that substantial amounts of material probably exist well beyond the boundaries of the reflection nebulosities. Indeed, the total mass in the cloud around GL2688 may equal that around IRC +10216 . More importantly, the clouds around both GL2688 and IRC +10216 are very rich in carbon; they are what we call carbon stars - relatively rare stars containing more carbon than oxygen rather than, as is typical, the reverse. More generally, most proto-planetary nebulae and, by extrapolation, planetary nebulae too are apparently carbon rich. These stars burn helium into carbon in their interiors and then expel the carbon into interstellar space, some day to be incorporated into new stars, planets and people. So much of the carbon in your scrambled eggs may have been cooked deep within objects like the Egg Nebula that lived, and died, many billions of years ago.

Where is this field heading now? Large new telescopes for IR and radio astronomy promise great advances and possibly some surprises in the ground-based study of cocoons around dying stars. But some of the most exciting discoveries should come after 1982 when NASA's infrared astronomical satellite is scheduled to be launched. The IRAS satellite will permit all-sky IR surveys at sensitivity levels much superior to all previous ground-based and rocket surveys. In addition to discovering more dying stars, IRAS should reveal numerous stars in the process of formation and even, perhaps, the IR radiation from a nearby super-advanced technical civilization (a Dyson sphere).

\section{Addendum}

The meeting report "Extragalactic jets: facts and fancies" (Nature 293, 336-337; 1981) was co-authored by Peter Scheuer and Mitchell Begelman. We apologize to $\mathrm{Dr}$ Begelman for omitting his name.

\title{
Radiative frustration by vacuum manipulation
}

\section{from Peter Knight}

TRANSITIONS between atomic states are induced by incident electromagnetic fields. Even in the absence of an incident field, the vacuum fluctuations and zero-point energy of the quantized field interact with atoms and are responsible for spontaneous emission and for small radiative corrections to atomic energy levels. Spontaneous emission can be viewed crudely as stimulated by the ground-state quantum fluctuations in the electromagnetic field coupled to the atom. Ordinary stimulated transitions can be altered by changing the incident field frequency or intensity, but spontaneous decay would appear to be unalterable unless the field vacuum state can be changed. Recently Kleppner of MIT proposed ${ }^{1}$ a method for doing just this, by placing the excited atom in a small high- $Q$ cavity which 'squeezes out' some of the radiation modes and changes the mode density. The cavity can either frustrate or enhance the decay by a factor of the order of $Q$ of the cavity, depending on the cavity detuning from atomic resonance ${ }^{2}$. The effect of mode confinement on decay lifetimes provides new and direct information on the nature of spontaneous decay.

The idea of altering the radiation field vacuum state is an old one, going back at least to work in the 1940 s on quantum Casimir forces between conducting plates. An enclosing cavity alters the boundary conditions of the problem and changes the appropriate mode expansion for the field. An elementary example of this is a onedimensional cavity supporting only standing waves: an excited atom in such a cavity will have a strongly position-dependent lifetime which is enhanced or diminished depending in part on whether the atom is sited at a node or antinode of the field mode. In the 1960s and 1970s, Barton, Power and others used appropriate mode expansions and counterterms to uncover cavity-mode alterations in atomic energies, radiative corrections and lifetimes. This extensive theoretical work on energy shifts has been supplemented by meagre and rather negative experimental activity (see, for example, ref.3).

Kleppner's suggestion, based in part on his Brandeis Summer Institute lectures of 1969 , is to 'freeze out' vacuum modes by using a waveguide cavity with characteristic dimensions small compared with the decay radiation wavelength. This seemed to be unattainable with optical frequencies involved in normal decay. The alteration of spontaneous decay rates by dielectric wave-

Peter Knight is in the Optics Section at the Blackett Laboratory, Imperial College, London. guide structures had already been discussed by Wittke ${ }^{4}$, with particular emphasis on its importance in heterojunction lightemitting diodes; Kleppner has reinvestigated waveguide effects but on highly excited Rydberg atoms. Such highly excited states decay slowly if they possess both a large principal quantum number and a large angular momentum; when they decay they do so slowly to a nearby Rydberg state, with the emission of very long-wavelength radiation. This enables larger and perhaps experimentally feasible waveguides to be used and Kleppner states that experiments are in progress.

More theoretical work will be needed before any experimental results can be properly evaluated. Kleppner concentrates solely on the 'mode density' and arrives at position-independent lifetimes from a modified golden rule. The atomic lifetime will clearly depend on the distance of the atom from the cavity wall simply because the 'inducing' vacuum field modes have a strong spatial dependence which is reflected in matrix elements; this has been omitted by Kleppner. His results seem to be an averaged-out approximation to the proper mode-expansion results and this needs examination. In particularly simple geometries (such as a pair of infinite mirrors separated by $L$, with an atom at position $Z$ from one of the mirrors), the cavity-dependent lifetimes can be computed using proper mode expansions ${ }^{5}$ or image techniques ${ }^{6}$. The effect of cavity wall conductivity has been investigated in such simple systems using various models (see, for example, refs 7 and 8).

It might be thought that experiments on these effects using optical decay wavelengths would be extraordinarily difficult, this is not the case: using the Langmuir-Blodgett monomolecular layer technique it is possible to situate fluorescent molecules a fixed and microscopic distance between dielectric or metallic surfaces and to study the alteration of spontaneous lifetimes by an altered local environment ${ }^{9}$. Such experiments have revealed that spontaneous decay is not an immutable effect but is sensitive to the details of the confining geometry, and Kleppner's work indicates fascinating modifications to the properties of highly excited atoms in optical waveguides.

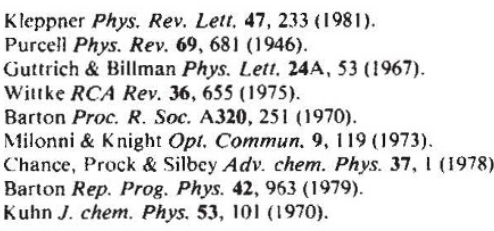

